\title{
SPHOTOM - Package for an Automatic Multicolour Photometry
}

\author{
Š. Parimucha ${ }^{1}$, M. Vaňko ${ }^{2}$, and P. Mikloš $\check{~}^{1}$ \\ ${ }^{1}$ Institute of Physics, University of P.J. Šafárik, Košice, Slovakia \\ email: stefan.parimucha@upjs.sk \\ ${ }^{2}$ Astronomical Institute of the Slovak Academy of Sciences, Tatranská Lomnica, Slovakia \\ email: vanko@ta3.sk
}

\begin{abstract}
We present basic information about package SPHOTOM for an automatic multicolour photometry. This package is in development for the creation of a photometric pipe-line, which we plan to use in the near future with our new instruments. It could operate in two independent modes, (i) GUI mode, in which the user can select images and control functions of package through interface and (ii) command line mode, in which all processes are controlled using a main parameter file. SPHOTOM is developed as a universal package for Linux based systems with easy implementation for different observatories. The photometric part of the package is based on the Sextractor code, which allows us to detect all objects on the images and perform their photometry with different apertures. We can also perform astrometric solutions for all images for a correct cross-identification of the stars on the images. The result is a catalogue of all objects with their instrumental photometric measurements which are consequently used for a differential magnitudes calculations with one or more comparison stars, transformations to an international system, and determinations of colour indices.
\end{abstract}

Keywords. photometry, CCD

The function of the SPHOTOM can be described in the following steps:

\section{Sorting}

It is the first step in command line mode, which creates different lists of images based on information in the FITS header of images as well as names of files. It uses a robust sorting scheme and it is written in Python using the PyFits module. The user can define types of lists, which will be used in next steps. Sorting could be executed also from GUI mode. For a correct functionality it is necessary to have a consistent FITS headers and/or image names, which are observatory dependent.

\section{Master images}

Create master images using an average or a median of input files. No other corrections are performed in this step. These images are stored in archive for a future use. The user can define a number of images entering into this procedure.

\section{Photometric reduction}

During photometric reduction of raw images we use created or archived master images. We can use, bias, dark frame, flat-field and dark for flat corrections. The procedure automatically controls image dimensions, temperatures and colours. The results are the lists of images based on their colours. 


\section{Photometry}

The photometric properties are derived from the reduced images using the Sextractor code (Bertin \& Arnouts 1996). We can control all photometry options using the parameters files of Sextrator (for more details see Sextrator manual). This package is very effective on uncrowded or semi-crowded fields and allows us to detect all objects above the defined background level on the images and perform their photometry with different user-defined apertures. It can also remove bad or corrupted detections (stars on the edges of images, saturated stars, cosmic ray hits). User can define different types of information in the output file.

\section{Identification}

If the FITS headers contain WCS (World Coordinate Systems) information (Calabretta \& Greisen, 2002), we perform cross-identification with external catalogs (USNO-A2.0 or Tycho) using up to 20 brightest stars on the image and the astrometric solution of all detected objects is calculated. If we have no WCS information we calculate an astrometric solution with known approximate coordinates of the image center and cross-identification with external catalogs. For each image, a file is created for the detected objects with their coordinates (celestial and image) and their instrumental magnitudes in apertures from photometry.

\section{Output catalogue}

After identification of the objects we have 2 possibilities:

(I) In GUI, the user can select up to 9 stars and generate their multicolour light curves with differential magnitudes. No other corrections are performed. The user can manually use procedures in the final corrections step.

(II) In command line mode, we generate differential magnitudes between all pairs of stars, create light curves and store them in a temporary database for an easier manipulation.

\section{Final corrections}

In the final step, we can perform several corrections:

1) Heliocentric correction of time.

2) Determination of extinction coefficients and reduction of systematic effects using SARS algorithm (Ofir et al., 2010).

3) Transformation to international system with known transformation coefficients.

4) Calculation of average comparison star from user selected objects.

Finally, we create the differential light curves.

Acknowledgements: This contribution was supported by VEGA project 2/0094/11.

\section{References}

Bertin, E. \& Arnouts, S., 1996, A\&AS, 117, 394

Calabretta, M. R. \& Greisen, E. W., 2002, A\&A, 395, 1077

Ofir et al., 2010, MNRAS (Letters), 404, L99 